\title{
Genetic Divergence Study in Sorghum (Sorghum bicolor L.) using $\mathrm{D}^{2}$ Analysis
}

\author{
P. Kavya ${ }^{1}$, V. Satyanarayana Rao ${ }^{2}$, B. Vijayalakshmi ${ }_{5}^{1}$, B. Sreekanth ${ }^{3}$, \\ Y. Radha Krishna ${ }^{4}$ and Nafeez Umar $^{5}$
}

${ }^{1}$ Department of GPBR, Agricultural College, ${ }^{3}$ Department of Crop Physiology, Agricultural College, ${ }^{4}$ Saline Water Scheme, College Farm, ${ }^{5}$ Department of Statistics and mathematics, Agricultural College, Bapatla, ANGRAU, A.P., India

${ }^{2} A D R$, Lam, Guntur, ANGRAU, A.P., India

*Corresponding author

\section{A B S T R A C T}

\begin{tabular}{|l|}
\hline Keyw or d s \\
Sorghum, D \\
statistics, Clusters, \\
Means
\end{tabular}

The study was undertaken to assess the genetic diversity among 110 sorghum genotypes using $\mathrm{D}^{2}$ statistics. 4 clusters were formed, highest no of genotypes are in cluster $-\mathrm{I}$ with 91, followed by cluster -II with 12, next by cluster-IV with 6 genotypes and cluster-III having solitary genotypes. The highest inter cluster distance was observed between cluster II \& IV (4018.74), Cluster II and III (2968.78), I and II (1050.81), I and IV (1332.68) and lowest between cluster III and IV (235.21). Cluster means for 9 characters were as following cluster I had high means for days to $50 \%$ flowering while cluster III recorded the lowest. As seen in the result of first character, cluster I showed low means for 1000 seed weight while cluster III has high means. Panicle weight recorded high mean in cluster IV and low in Cluster I. seed yield/plant has observed high means in cluster I and low means in cluster III. The genotypes in cluster -II and Cluster-III are divergent according to this study and hybridisation programme between these genotype clusters would be rewarding with heterotic response from its progeny. Based on the mean values no single genotype possessed all desirable characters, most yield and yield related attributes is-2834 performed for biomass type's NSJB-6652.

\section{Introduction}

Sorghum, a $\mathrm{C}_{4}$ crop belonging to Poaceae family is a food crop in African and Indian contexts while used as feeds stock I European and western countries, besides the food serving purpose it is used as fodder as well as biofuel purpose. It provides the minimum guaranteed yields as it can thrive the hard drought situations (Aruna et al., 2011). The origin of sorghum is Ethiopia (Wetand Huckabay, 1967) of Africa where wide diversity of germplasm is available along with India, possessing great diversity of germplasm particularly in arid and semi-arid tropics maintained by ICRISAT and IIMR Institutions. To enhance the yield of the present day cultivars best parents are to be 
identified from the existing germplasm by studying the diversity among them.

The genetic diversity may arise due to geographical blocks or due to cross ability in the cultivated and wild species of crop. More chance of heterotic groups is performed if diversity is broad and breeding for biotic and abiotic resistances will be productive (Elangovan and Babu, 2015). This is the conventional method based on the morphological traits irrespective of the laboratory preparations (Ahalawat et al., 2018) (Prasanna, 2010). The present investigation was undertaken to study the divergence present in the germplasm using $\mathrm{D}^{2}$ analysis which was given by Mahalanobis (1936). $\mathrm{D}^{2}$ is one of the reliable methods to understand the diversity using tochers method.

\section{Materials and Methods}

110 sorghum genotypes were analysed in RBD with three replications at Agricultural college farm, Bapatla during Kharif, 2017. Each and every genotype was planted in $2 \mathrm{~m}$ row, with the spacing of $45 \times 15 \mathrm{~cm}$. All the recommended cultural practices were followed according to the crop needs. The data was collected on the following observations days to $50 \%$ flowering, days to maturity, plant height, stem girth, no of nodes, panicle weight, 1000 grain weight, seed yield/ plant, stalk yield/plant.

\section{Results and Discussion}

The present $\mathrm{D}^{2}$ was applied to 110 genotypes which classified the total genotypes into 4 clusters. Cluster $-\mathrm{I}$ with highest number of genotypes i.e., 91, followed by cluster -II with 12 genotypes and cluster -IV with 06 genotypes and cluster-III is a solitary one (Table 1). The results are in accordance with Patankar et al., (2005), Rohman et al., (2004) and Shinde et al., (2013). Inter cluster values are higher than intra cluster values as observed by Ahalawat et al., (2018), Prasad et al., (2017) earlier in their studies suggesting wide diversity among the genotypes. The highest inter cluster distance was observed between cluster II and IV (4018.74), Cluster II and III (2968.78), I and II (1050.81), I and IV (1332.68) and lowest between cluster III and IV (235.21). The crossing among cluster II and IV breeds the most transgressive segregants when planned for hybridisation programme. The least inter cluster distance between III and IV indicates close association between the genotypes which is not desirable for hybridisation programme. The maximum intra cluster distance was observed between IV (402.28) and cluster I (254.53) possessing high diversity would produce segregants of high genetic advance and least intra cluster distance observed or cluster II indicating homogenous populations and not reliable for hybridisation programme. The intra and inter cluster values were given in table 2 .

The results of cluster means for 9 characters were as following cluster I had high means for days to $50 \%$ flowering while cluster III recorded the lowest, days to maturity has high means in cluster II and low in Cluster IV, no of nodes per plant and plant height had high means in cluster III low in cluster II and IV respectively. Cluster II poses high means for stalk yield per plant while cluster -III has reverse version. Character stem girth shown low means in cluster IV and high means for cluster II. As seen in the result of first character, cluster I showed low means for 1000 seed weight while cluster III has high means. Panicle weight recorded high mean in cluster IV and low in Cluster I. seed yield/plant has observed high means in cluster I and low means in cluster III (Table 3).

The genotypes in cluster -II (NSJB-6629, EC23, EP-84, CJV-24, CJV-18, NSJB-6585, 
SCVS-29, EG-74, EG-39, NSJB-6648, GGUB-63, GGUB-62) and Cluster-III (EB22) are divergent according to this study and

hybridisation programme between these genotype clusters would be rewarding with heterotic response from its progeny (Table 4).

Table.1 grouping of genotypes according to clusters

\begin{tabular}{|c|c|c|}
\hline Cluster & $\begin{array}{c}\text { No of } \\
\text { Genotypes }\end{array}$ & Genotypes List \\
\hline I & 91 & $\begin{array}{l}\text { CJV-07, SPV-2328, GGUB-45, ICSV-12012, SECS-28, RAJ-9, } \\
\text { EG-24, IS-1331, EP-29, SSS-15, IS-2834, IS-29650, EC-45, } \\
\text { ICSV-25306, ICSV-25316,E-40, CJV-24, EB-20, PV-22, EG-83, } \\
\text { IS-27239, SSS-46, SSS-10, GGUB-28, IS-29469, CJV-25, SEVS- } \\
\text { 20, EG-80, IS-3980, NSJB-6577, GGUB-29, SSS-84, GGUB-64, } \\
\text { NSJB-6662, PHULE VASHUNHARA, GGUB-13, EG-23, } \\
\text { GGUB-68, GGUB-61, E-63, SPV-2326, RSSV-1381, IS-27072, } \\
\text { IS-30310, EC-25, SSS-14, CSV-24SS, SSS-62, GGUB-65, EP-80, } \\
\text { EB-14, GGUB-50, SSS-65, IS-2814,CJV-16, NSJB-6657,IS- } \\
\text { 29308, EG-22, GGUB-27, SPV-2196, ICSSH-71, NSJB-6629, } \\
\text { ICSV-25308, CJV-26, NSJB-6605, EB-15, GGUB-43, SPV- } \\
\text { 2327, SSS-74, ICSV-15006, EC-20, SSS-23, CJV-17, IS-4599, } \\
\text { DHBM-3, SEVS-04, SSS-49, CJV-19, EG-82, GGUB-67, PV-12, } \\
\text { CSV-19SS, EG-25, EG-11, IS-2337, Ep-61, CJV-21, IS-3515, } \\
\text { EG-21, EB-19, GGUB-33 }\end{array}$ \\
\hline II & 12 & $\begin{array}{l}\text { NSJB-6629, EC-23, EP-84, CJV-24, CJV-18, NSJB-6585, SCVS- } \\
\text { 29, EG-74, EG-39, NSJB-6648, GGUB-63, GGUB-62 }\end{array}$ \\
\hline III & 01 & EB-22 \\
\hline IV & 06 & IS-7474, EG-19, IS-6910,POP-15,EG-78,GGUB-45 \\
\hline
\end{tabular}

Table.2 Intra and Inter clusters D value and extent of diversity among the clusters

\begin{tabular}{|l|c|c|c|c|}
\hline Clusters & I & II & III & IV \\
\hline I & 254.93 & 1050.81 & 730.03 & 1332.68 \\
\hline II & & 130.21 & 2968.78 & 4018.74 \\
\hline III & & & 0.00 & 235.21 \\
\hline IV & & & & 402.28 \\
\hline
\end{tabular}

Table.3 Mean values of clusters for 9 characters in 110 sorghum genotypes (Tocher's method)

\begin{tabular}{|l|l|l|l|l|l|l|l|l|l|}
\hline Clusters DAF 50\% & DM & N.D & P.H & S.G & 1000 S.W & P.W & STK/P & SY/P \\
\hline I & $\mathbf{7 9 . 6 0}$ & 112.48 & 13.82 & 372.41 & 1.90 & $\mathbf{2 9 . 2 2}$ & $\mathbf{7 2 . 1 9}$ & 604.55 & 44.00 \\
\hline II & 97.39 & $\mathbf{1 4 3 . 1 9}$ & $\mathbf{1 5 . 6 7}$ & 392.47 & $\mathbf{2 . 0 3}$ & 29.67 & 74.77 & $\mathbf{6 7 4 . 6 2}$ & 41.77 \\
\hline III & $\mathbf{5 5 . 0 0}$ & 87.00 & $\mathbf{1 1 . 0 0}$ & $\mathbf{4 3 1 . 7 4}$ & 1.93 & $\mathbf{3 1 . 3 6}$ & 73.00 & $\mathbf{4 3 0 . 8 0}$ & $\mathbf{3 9 . 9 4}$ \\
\hline IV & 66.33 & $\mathbf{7 9 . 5 0}$ & 11.83 & $\mathbf{3 2 0 . 4 5}$ & $\mathbf{1 . 7 6}$ & 29.63 & $\mathbf{7 8 . 3 4}$ & 590.01 & $\mathbf{4 4 . 5 7}$ \\
\hline
\end{tabular}

DAF $50 \%=$ Days to $50 \%$ flowering, DM: Days to maturity, N.O.N: No of nodes, P.H: Plant height, S.G: Stem girth, 1000 S.W: 1000 Seed weight, P.W: Panicle weight, SY/P: See yield/plant, STKY/P: Stalk yield /plant 
Table.4 Means values for 9 characters in 110 sorghum genotypes

\begin{tabular}{|c|c|c|c|c|c|c|c|c|c|c|}
\hline S.NO & GENOTYPE & $\begin{array}{l}\text { DAF } \\
50 \%\end{array}$ & DM & N.O.N & P.H & S.G & $\begin{array}{l}10000 \\
\text { G.W }\end{array}$ & P.W & SY/P & STKY/P \\
\hline 1 & Cjv-07 & 74.0 & 105.7 & 16.0 & 457.7 & 1.2 & 25.2 & 74.7 & 46.7 & 880.8 \\
\hline 2 & Spv-2328 & 70.7 & 95.7 & 11.7 & 357.2 & 1.4 & 29.5 & 84.0 & 52.3 & 236.0 \\
\hline 3 & GGUB-45 & 66.7 & 91.0 & 11.7 & 336.5 & 1.6 & 31.6 & 93.3 & 61.0 & 239.5 \\
\hline 4 & ICSV-12012 & 80.7 & 109.7 & 13.0 & 334.2 & 1.9 & 31.1 & 85.3 & 52.0 & 410.3 \\
\hline 5 & NSJB-6585 & 110.3 & 145.3 & 22.0 & 462.9 & 1.2 & 27.3 & 75.3 & 48.3 & 989.0 \\
\hline 6 & SEVS-28 & 73.0 & 110.7 & 13.0 & 374.0 & 1.9 & 20.6 & 67.3 & 33.3 & 649.5 \\
\hline 7 & RAJ-9 & 68.7 & 112.3 & 14.3 & 345.1 & 1.8 & 26.2 & 84.7 & 45.3 & 601.5 \\
\hline 8 & EG-24 & 71.7 & 112.0 & 13.7 & 416.0 & 1.8 & 27.0 & 73.3 & 39.0 & 435.8 \\
\hline 9 & IS-1331 & 71.3 & 114.3 & 14.0 & 296.8 & 1.5 & 35.7 & 76.3 & 48.3 & 420.3 \\
\hline 10 & EP-29 & 71.7 & 119.3 & 13.0 & 191.7 & 1.6 & 23.0 & 68.7 & 47.7 & 320.7 \\
\hline 11 & SSS-15 & 72.0 & 107.3 & 17.7 & 526.1 & 1.7 & 29.2 & 81.3 & 61.0 & 1132.3 \\
\hline 12 & IS-2834 & 73.0 & 112.3 & 11.7 & 325.7 & 0.3 & 46.6 & 111.0 & 68.0 & 149.7 \\
\hline 13 & IS-29650 & 54.0 & 89.7 & 10.0 & 226.1 & 0.5 & 38.8 & 74.3 & 42.3 & 208.7 \\
\hline 14 & EC-45 & 67.3 & 104.3 & 14.7 & 428.7 & 1.8 & 31.1 & 79.7 & 48.3 & 445.2 \\
\hline 15 & IS-6910 & 54.7 & 85.3 & 4.3 & 182.6 & 1.9 & 28.7 & 91.0 & 45.3 & 576.6 \\
\hline 16 & POP-15 & 54.0 & 82.3 & 13.0 & 421.3 & 2.0 & 34.5 & 90.0 & 50.0 & 470.0 \\
\hline 17 & ICSV-25306 & 90.3 & 135.3 & 13.3 & 379.5 & 1.5 & 28.7 & 89.7 & 46.3 & 479.7 \\
\hline 18 & EC-23 & 112.7 & 140.7 & 14.0 & 397.7 & 1.7 & 34.3 & 87.0 & 50.7 & 493.7 \\
\hline 19 & ICSV-25316 & 80.0 & 120.7 & 14.0 & 368.6 & 1.7 & 24.2 & 72.3 & 32.3 & 987.1 \\
\hline 20 & E-40 & 90.0 & 135.0 & 12.7 & 376.3 & 1.6 & 33.1 & 86.0 & 38.7 & 523.3 \\
\hline 21 & CJV-24 & 101.7 & 143.3 & 18.3 & 378.9 & 1.7 & 35.7 & 87.0 & 45.0 & 483.6 \\
\hline 22 & EB-20 & 75.3 & 113.3 & 11.0 & 340.9 & 1.9 & 29.8 & 85.7 & 45.3 & 447.8 \\
\hline 23 & PV-22 & 88.0 & 118.7 & 11.0 & 334.2 & 1.1 & 32.5 & 80.7 & 49.0 & 356.3 \\
\hline 24 & EG-83 & 115.3 & 142.3 & 19.7 & 442.3 & 2.2 & 26.7 & 64.0 & 36.0 & 979.3 \\
\hline 25 & IS-27239 & 72.0 & 105.0 & 15.0 & 401.0 & 2.0 & 32.8 & 72.3 & 42.3 & 833.5 \\
\hline 26 & SSS-46 & 86.7 & 118.0 & 16.0 & 429.3 & 1.9 & 33.2 & 86.7 & 35.7 & 726.4 \\
\hline 27 & EG-78 & 87.3 & 116.0 & 15.7 & 258.9 & 2.0 & 20.1 & 44.7 & 22.3 & 753.9 \\
\hline 28 & SSS-10 & 87.0 & 113.7 & 15.0 & 437.7 & 1.9 & 35.7 & 62.7 & 29.0 & 586.0 \\
\hline 29 & GGUB-28 & 57.0 & 83.0 & 17.0 & 119.7 & 1.8 & 37.7 & 76.3 & 41.7 & 491.9 \\
\hline 30 & IS-29469 & 64.7 & 95.0 & 8.7 & 211.3 & 1.3 & 22.7 & 69.0 & 50.3 & 163.4 \\
\hline 31 & CJV-25 & 78.0 & 120.3 & 11.0 & 224.7 & 1.9 & 34.6 & 81.0 & 58.7 & 213.3 \\
\hline 32 & SEVS-20 & 71.0 & 101.3 & 11.7 & 295.9 & 0.4 & 38.2 & 86.3 & 55.7 & 253.4 \\
\hline 33 & GGUB-62 & 82.0 & 111.0 & 13.7 & 395.8 & 2.1 & 40.4 & 90.0 & 51.0 & 489.1 \\
\hline 34 & IS-7474 & 68.0 & 111.0 & 13.0 & 347.5 & 1.8 & 34.4 & 76.3 & 45.7 & 775.2 \\
\hline 35 & EG-80 & 111.0 & 159.0 & 16.7 & 434.6 & 1.9 & 33.1 & 76.0 & 36.0 & 876.7 \\
\hline 36 & IS-3980 & 26.3 & 54.3 & 12.0 & 347.7 & 1.6 & 29.5 & 68.0 & 27.0 & 235.8 \\
\hline 37 & EP-84 & 76.0 & 108.0 & 9.0 & 257.9 & 1.7 & 32.6 & 79.0 & 33.3 & 240.8 \\
\hline 38 & EG-19 & 68.0 & 96.0 & 13.3 & 385.9 & 1.6 & 28.5 & 72.0 & 40.3 & 806.3 \\
\hline 39 & NSJB-6577 & 104.7 & 141.0 & 17.7 & 451.9 & 1.6 & 21.1 & 45.3 & 26.7 & 622.3 \\
\hline 40 & GGUB-29 & 55.0 & 81.0 & 9.0 & 282.1 & 1.9 & 34.8 & 59.0 & 28.3 & 264.0 \\
\hline 41 & SSS-84 & 70.0 & 101.3 & 15.0 & 334.3 & 1.9 & 31.3 & 78.3 & 29.7 & 560.6 \\
\hline
\end{tabular}




\begin{tabular}{|c|c|c|c|c|c|c|c|c|c|c|}
\hline 42 & GGUB-64 & 86.0 & 104.7 & 14.0 & 377.0 & 1.7 & 27.5 & 71.7 & 38.3 & 636.3 \\
\hline 43 & NSJB-6662 & 109.7 & 123.3 & 20.7 & 480.7 & 1.6 & 22.9 & 69.0 & 36.7 & 883.7 \\
\hline 44 & $\begin{array}{l}\text { PHULE } \\
\text { VASHUNDRA }\end{array}$ & 113.3 & 135.7 & 20.0 & 510.8 & 1.9 & 30.6 & 71.0 & 55.0 & 1196.7 \\
\hline 45 & GGUB-13 & 84.3 & 113.7 & 14.7 & 414.7 & 2.1 & 29.1 & 72.3 & 44.0 & 670.3 \\
\hline 46 & EG-23 & 73.7 & 104.0 & 14.0 & 403.0 & 2.1 & 28.5 & 80.0 & 52.3 & 774.6 \\
\hline 47 & GGUB-68 & 73.0 & 104.7 & 17.3 & 499.5 & 1.5 & 35.2 & 77.0 & 47.0 & 497.7 \\
\hline 48 & GGUB-61 & 86.0 & 113.0 & 14.0 & 434.5 & 1.8 & 38.1 & 80.7 & 56.3 & 694.2 \\
\hline 49 & E-63 & 87.7 & 115.3 & 16.0 & 412.1 & 1.8 & 34.5 & 66.7 & 44.3 & 1158.0 \\
\hline 50 & SPV-2326 & 73.7 & 109.0 & 12.3 & 361.2 & 1.1 & 26.9 & 62.3 & 50.7 & 886.7 \\
\hline 51 & RSSV-1381 & 81.7 & 117.0 & 12.0 & 315.3 & 1.3 & 31.4 & 73.7 & 55.3 & 706.3 \\
\hline 52 & IS-27072 & 112.7 & 124.7 & 20.3 & 457.7 & 1.9 & 32.3 & 73.3 & 46.0 & 798.3 \\
\hline 53 & IS-30310 & 66.3 & 94.0 & 10.3 & 117.7 & 2.2 & 29.7 & 64.7 & 55.3 & 686.7 \\
\hline 54 & EC-25 & 78.7 & 110.0 & 15.0 & 367.5 & 2.1 & 23.2 & 60.3 & 47.0 & 452.3 \\
\hline 55 & SSS-14 & 83.7 & 119.0 & 15.0 & 395.2 & 2.5 & 31.5 & 69.0 & 62.7 & 920.3 \\
\hline 56 & CSV-24SS & 84.0 & 120.3 & 13.7 & 357.1 & 2.3 & 33.4 & 79.0 & 57.0 & 704.8 \\
\hline 57 & SSS-62 & 88.3 & 117.0 & 17.0 & 390.2 & 2.2 & 31.8 & 73.3 & 58.3 & 671.2 \\
\hline 58 & GGUB-65 & 89.7 & 120.0 & 15.3 & 415.6 & 2.8 & 38.0 & 72.0 & 59.0 & 594.3 \\
\hline 59 & EP-80 & 85.0 & 109.0 & 15.3 & 412.3 & 3.5 & 30.5 & 76.7 & 62.0 & 1136.3 \\
\hline 60 & EB-14 & 69.0 & 106.0 & 14.3 & 409.9 & 2.2 & 19.1 & 42.3 & 26.7 & 505.0 \\
\hline 61 & GGUB-50 & 92.3 & 124.3 & 14.0 & 383.9 & 2.6 & 23.1 & 52.3 & 32.3 & 473.5 \\
\hline 62 & SCVS-29 & 77.0 & 101.0 & 14.0 & 372.4 & 1.8 & 24.5 & 60.7 & 42.0 & 909.3 \\
\hline 63 & SSS-65 & 96.0 & 141.0 & 13.7 & 374.4 & 1.6 & 30.0 & 69.7 & 38.3 & 857.4 \\
\hline 64 & IS-2814 & 74.7 & 114.0 & 16.3 & 241.6 & 2.2 & 34.0 & 73.3 & 47.7 & 652.3 \\
\hline 65 & CJV-16 & 93.0 & 119.7 & 19.0 & 471.5 & 2.5 & 28.8 & 81.0 & 52.0 & 1036.7 \\
\hline 66 & CJV-18 & 97.0 & 125.0 & 13.0 & 410.5 & 2.7 & 31.7 & 82.3 & 36.7 & 691.5 \\
\hline 67 & NSJB-6652 & 110.7 & 141.0 & 21.7 & 470.2 & 3.7 & 26.8 & 74.0 & 39.3 & 1334.8 \\
\hline 68 & IS-29008 & 95.0 & 114.7 & 13.3 & 389.1 & 1.8 & 33.1 & 66.3 & 21.3 & 280.3 \\
\hline 69 & EG-22 & 66.3 & 93.3 & 12.3 & 334.0 & 1.7 & 19.7 & 42.3 & 21.7 & 269.1 \\
\hline 70 & GGUB-27 & 98.0 & 129.0 & 11.7 & 416.9 & 1.9 & 23.8 & 50.3 & 30.7 & 440.6 \\
\hline 71 & ICSSH-71 & 97.0 & 125.0 & 14.3 & 340.0 & 2.2 & 34.2 & 72.3 & 38.7 & 658.6 \\
\hline 72 & EG-74 & 86.7 & 115.3 & 12.0 & 447.0 & 2.1 & 25.0 & 63.7 & 30.3 & 645.4 \\
\hline 73 & NSJB-6629 & 112.3 & 138.0 & 18.0 & 443.1 & 3.0 & 32.6 & 80.0 & 51.0 & 821.7 \\
\hline 74 & CJV-24 & 92.7 & 124.7 & 13.0 & 418.3 & 1.9 & 29.7 & 78.0 & 51.3 & 709.9 \\
\hline 75 & GGUB-63 & 112.3 & 152.0 & 15.0 & 410.8 & 2.1 & 37.3 & 81.0 & 58.0 & 585.3 \\
\hline 76 & ICSV-25306 & 96.7 & 133.0 & 13.0 & 396.3 & 2.1 & 28.7 & 72.3 & 46.0 & 789.0 \\
\hline 77 & CJV-26 & 66.0 & 93.0 & 12.7 & 327.4 & 1.8 & 22.9 & 70.7 & 49.3 & 378.8 \\
\hline 78 & NSJB-6605 & 85.0 & 124.7 & 16.3 & 386.4 & 2.3 & 33.3 & 79.0 & 53.0 & 411.4 \\
\hline 79 & SPV-2196 & 96.7 & 124.7 & 14.3 & 433.1 & 2.2 & 30.1 & 79.3 & 37.3 & 717.2 \\
\hline 80 & EB-15 & 91.7 & 117.7 & 11.3 & 414.6 & 1.3 & 28.0 & 65.3 & 48.0 & 799.6 \\
\hline 81 & GGUB-43 & 84.7 & 127.0 & 15.0 & 410.5 & 2.1 & 26.0 & 61.3 & 40.3 & 659.7 \\
\hline 82 & EB-22 & 55.3 & 87.0 & 11.0 & 431.7 & 1.9 & 31.4 & 72.7 & 39.3 & 430.8 \\
\hline 83 & SPV-2327 & 83.0 & 116.7 & 13.0 & 386.3 & 1.8 & 25.4 & 66.7 & 39.0 & 395.0 \\
\hline 84 & SSS-74 & 70.0 & 104.7 & 15.0 & 372.9 & 2.0 & 30.3 & 57.7 & 20.7 & 916.0 \\
\hline
\end{tabular}




\begin{tabular}{|c|c|c|c|c|c|c|c|c|c|c|}
\hline 85 & ICSV-15006 & 92.3 & 116.7 & 13.0 & 352.6 & 1.7 & 29.4 & 73.7 & 44.7 & 506.9 \\
\hline 86 & EC-20 & 87.3 & 116.0 & 12.3 & 441.9 & 2.2 & 32.0 & 78.7 & 51.0 & 503.8 \\
\hline 87 & SSS-23 & 91.0 & 120.0 & 12.0 & 457.9 & 2.2 & 25.5 & 81.0 & 60.0 & 416.3 \\
\hline 88 & CJV-17 & 81.3 & 124.0 & 14.3 & 306.0 & 2.7 & 38.2 & 76.0 & 46.0 & 488.5 \\
\hline 89 & IS-4599 & 56.7 & 93.0 & 12.0 & 395.8 & 1.7 & 24.0 & 82.3 & 54.7 & 454.5 \\
\hline 90 & NSJB-6648 & 110.0 & 141.0 & 19.7 & 271.0 & 1.9 & 16.7 & 48.3 & 25.0 & 861.0 \\
\hline 91 & EG-39 & 90.3 & 138.0 & 13.3 & 453.0 & 1.8 & 21.4 & 54.7 & 35.0 & 420.0 \\
\hline 92 & HВМ-3 & 57.0 & 121.7 & 12.7 & 434.3 & 1.9 & 35.4 & 65.3 & 40.7 & 442.9 \\
\hline 93 & SEVS-04 & 72.3 & 108.0 & 13.3 & 393.5 & 2.0 & 28.4 & 56.3 & 30.0 & 437.7 \\
\hline 94 & GGUB-54 & 56.3 & 91.3 & 12.3 & 368.2 & 2.0 & 26.5 & 61.0 & 41.0 & 530.0 \\
\hline 95 & SSS-49 & 106.0 & 113.0 & 13.3 & 353.2 & 2.8 & 32.7 & 55.0 & 36.7 & 405.7 \\
\hline 96 & CJV-19 & 81.7 & 107.3 & 16.0 & 418.3 & 1.9 & 21.1 & 59.3 & 33.0 & 619.1 \\
\hline 97 & EG-82 & 82.0 & 113.0 & 15.3 & 445.4 & 2.3 & 17.0 & 47.7 & 26.0 & 867.4 \\
\hline 98 & GGUB-67 & 101.3 & 119.0 & 13.0 & 374.5 & 1.9 & 19.1 & 66.3 & 40.0 & 926.7 \\
\hline 99 & PV-12 & 87.0 & 106.7 & 13.0 & 396.1 & 2.0 & 31.0 & 91.0 & 68.0 & 513.0 \\
\hline 100 & EG-11 & 58.7 & 111.0 & 12.3 & 414.3 & 1.2 & 28.5 & 61.0 & 32.7 & 404.3 \\
\hline 101 & IS-2337 & 68.7 & 109.0 & 14.7 & 424.7 & 1.8 & 32.0 & 86.0 & 52.3 & 422.7 \\
\hline 102 & EG-25 & 61.3 & 108.0 & 14.3 & 422.3 & 1.3 & 28.7 & 80.0 & 49.3 & 623.3 \\
\hline 103 & CSV-19SS & 69.0 & 105.0 & 10.7 & 346.1 & 1.3 & 29.7 & 73.0 & 47.3 & 325.7 \\
\hline 104 & EP-61 & 68.7 & 105.0 & 16.7 & 341.0 & 2.3 & 29.9 & 67.0 & 43.0 & 714.3 \\
\hline 105 & CSH-22SS & 69.0 & 105.0 & 13.3 & 349.7 & 2.6 & 34.5 & 71.3 & 51.0 & 1093.0 \\
\hline 106 & EB-19 & 78.7 & 120.7 & 14.3 & 391.9 & 2.2 & 16.0 & 64.0 & 29.3 & 944.0 \\
\hline 107 & GGUB-33 & 84.3 & 121.7 & 14.7 & 411.5 & 2.2 & 14.5 & 70.0 & 42.3 & 963.7 \\
\hline 108 & EG-21 & 60.3 & 92.3 & 12.0 & 315.6 & 2.2 & 22.8 & 74.0 & 39.7 & 803.9 \\
\hline 109 & IS-3515 & 81.3 & 102.3 & 11.3 & 315.9 & 2.3 & 30.9 & 69.3 & 51.3 & 587.4 \\
\hline \multirow[t]{10}{*}{110} & CJV-21 & 76.3 & 104.7 & 13.0 & 387.3 & 1.9 & 27.3 & 73.0 & 50.7 & 412.3 \\
\hline & MEAN & 80.89 & 113.80 & 13.95 & 372.30 & 1.91 & 29.31 & 72.81 & 43.75 & $609 . .8$ \\
\hline & C.V & 9.62 & 0.78 & 14.61 & 11.45 & 15.62 & 6.02 & 2.30 & 5.69 & 16.90 \\
\hline & F Ratio & 13.07 & 1007.63 & 5.81 & 9.51 & 7.7 & 31.47 & 147.78 & 50.78 & 17.50 \\
\hline & F prob & 0.00 & 0.00 & 0.00 & 0.00 & 0.00 & 0.00 & 0.00 & 0.00 & 0.00 \\
\hline & S.E & 4.47 & 0.51 & 1.17 & 24.63 & 0.17 & 1.02 & 0.96 & 1.43 & 59.53 \\
\hline & C.D 5\% & 12.48 & 1.43 & 3.28 & 68.65 & 0.48 & 2.84 & 2.69 & 4.01 & 165.93 \\
\hline & C.D $1 \%$ & 16.45 & 1.89 & 4.32 & 90.52 & 0.63 & 3.74 & 3.55 & 5.28 & 218.78 \\
\hline & Range Lowest & 26.33 & 54.33 & 4.33 & 117.66 & 0.34 & 14.53 & 42.35 & 20.99 & 149.70 \\
\hline & Range highest & 115.33 & 159 & 22.00 & 526.10 & 3.70 & 46.62 & 111.30 & 68.50 & 1334.81 \\
\hline
\end{tabular}

DAF $50 \%=$ Days to $50 \%$ flowering, DM: Days to maturity, N.O.N: No of nodes, P.H: Plant height, S.G: Stem girth, 1000 S.W: 1000 Seed weight, P.W: Panicle weight, SY/P: See yield/plant, STKY/P: Stalk yield/plant

\section{Range, means of the genotypes}

\section{Days to $50 \%$ flowering}

Means for days to $50 \%$ flowering ranges from 26 to 115 with mean of 80.89 days.

The genotype IS-3980 and highest is in genotype EG-83.

\section{Days to maturity}

Maximum no of days for maturity recorded for genotype EG-80 while minimum were recorded for is-3980 the early flowering genotype with average days of 113.80 near to 4 months. 


\section{Number of nodes}

High no of nodes (22) for NSJB-6585 while less no of nodes (4.33) IS-6910 with mean of 13.95

\section{Plant height}

IS-30310 recorded the lowest height 117.66, while SSS-15 recorded the highest 526.10, while the average height measured is 372.50 .

\section{Stem girth}

Lowest range for stem girth is IS-2834(0.3) highest for NSJB-6652(3.70) with mean of 1.91 .

\section{0 grain weight}

IS-2834 recorded high value of 46.62, GGUB-33 recorded 14.53 with a mean of 29.31 .

\section{Panicle weight}

High weighed panicle among the genotypes is IS-2834, and low weighed is eb-14.

\section{Seed yield/plant}

Lowest yield was recorded for SSS-74, while high yield is recorded for two genotypes pv12. Is-2834 with average yield of 43.75 .

\section{Stalk yield/plant}

NSJB-6652 recorded as high biomass type having (1334.81) of stalk weight while lowest is IS-2834 (149.70) with mean of 609.8.

Based on the mean values no single genotype possessed all desirable characters, for most yield and yield related attributes IS-2834 performed well and for biomass type's it is NSJB-6652.

\section{References}

Ahalawat, N.K., Arya, V.K., Kumar, P and Singh, S.K. 2018. Genetic divergence in forage sorghum (Sorghum bicolor L. Moench). Journal of Applied and Natural Science, 10(1): 439-444.

Aruna, C., Bhagwat, V.R., Madhusudhana, R., Vittal Sharma, Hussain, T., Ghorade, R.B., Khandalkar, H.G., Audilakshmi, S. and Seetharama, N. 2011. Identification and validation of genomic regions that affect shootfly resistance in sorghum [Sorghum bicolor (L.) Moench]. Theory Applied Genetics. 122: $1617-1630$.

Elangovan, M. and Babu, P. K. (2015). Genetic variability and diversity of sorghum land race collected from Uttar Pradesh India. Indian Journal of plant genetic resources. 28(2): 21322.

Mahalanobis, P. C. (1936), "On the generalized distance in statistics," Proceedings of the National Institute of Sciences of India, 12: 49-55.

Patankar, A. B., Sonone, A. H., Patil, J. V. and Sarode, N. D. 2005. Genetic divergence in Sweet Sorghum. J. of Maharashtra Agric. Univ. 30(2): 175177.

Prasad, B.H.V. and Biradar, B.D. 2017. Genetic Diversity Studies in Minicore Collection of Rabi Sorghum [Sorghum bicolor. (L)] Using $\mathrm{D}^{2}$ Statistics. Int.J.Curr.Microbiol.App.Sci. 6(7): 850856.

Prasanna, B.M. (2010). Phenotypic and molecular diversity of maize landraces: characterization and utilization. The Indian Journal of Genetics and Plant Breeding. 70: 315-327.

Rohman, M.M., Hakim, M.A., Sultana, N.A., Kabir, M.E., Hasanuzzan and Ali, M. 2004. Genetic Divergence Analysis in Sorghum (Sorghum bicolor L.). Asian Journal of Plant Sciences, 3: 211-214. 
Shinde, D., Chavan, S. and Jadhav, B.D. 2013. Study of genetic divergence in sweet sorghum [Sorghum bicolor (L.) Moench]. The Bioscan. 8(1). 135-138.
Wet, J. M. J. de. and Huckabay, J. P. 1967. The Origin of Sorghum bicolor. II. Distribution and Domestication. Evolution. 21(4): 87-802.

\section{How to cite this article:}

Kavya, P., V. Satyanarayana Rao, B. Vijayalakshmi, B. Sreekanth, Y. Radha Krishna and Nafeez Umar. 2019. Genetic Divergence Study in Sorghum (Sorghum bicolor L.) using D ${ }^{2}$ Analysis. Int.J.Curr.Microbiol.App.Sci. 8(06): 24-31. doi: https://doi.org/10.20546/ijcmas.2019.806.004 